\title{
Diferentes Perspectivas nas Abordagens da Saúde/ Doença
}

\section{Different Perspectives in Health/IIIness Approaches}

\section{Adélcio Machado dos Santos}

Doutor em Engenharia e Gestão do Conhecimento pela Universidade Federal de Santa Catarina (UFSC). Pós-Doutor em Gestão do Conhecimento pela UFSC Universidade do Alto Vale do Rio do Peixe (UNIARP).

Endereço: Rua Victor Baptista Adami, n 800 - Centro - Caçador/SC/Brasil CEP 89500-199

E-mail: adelciomachado@gmail.com

\section{RESUMO}

O homem é visto num aspecto mais abrangente como sendo um cientista imperfeito, inserido numa realidade social, os sujeitos e seus laços se estabelecem em um território na busca de qualidade de vida. O objetivo do estudo é investigar as diferentes perspectivas elaboradas na abordagem do binômio saúde e doença. Embora sejam muito diversas em razão da peculiaridade de seus contextos culturais, as sociedades apresentam, também, características comuns. Necessita do desempenho de certas funções universais, indispensáveis ao prosseguimento de seu curso, entre elas a saúde. É bem verdade que estar saudável vai além de não ser acometido por um tipo de enfermidade, relacionada a aspectos como cultura, meio ambiente, congênito, genético, entre outros, ligados à história de cada indivíduo. É por essa razão que a educação em saúde deve ser o objetivo dos profissionais da saúde para o indivíduo, para o melhor da coletividade.

Palavras-chave: Saúde, Doença, Educação, Abordagem.

\section{ABSTRACT}

Man is seen in a broader aspect as being an imperfect scientist, inserted in a social reality, the subjects and their bonds are established in a territory in the search for quality of life. The objective of the study is to investigate the different perspectives elaborated in the approach to the binomial health and disease. Although they are very diverse due to the peculiarity of their cultural contexts, societies also have common characteristics. It requires the performance of certain universal functions, indispensable to the continuation of its course, among them health. It is very true that being healthy goes beyond not being affected by a type of illness, related to aspects such as culture, environment, congenital, genetic, among others, linked to the history of each individual. It is for this reason that health education should be the goal of health professionals for the individual, for the best of the collectivity.

Keywords: Health, Disease, Education, Approach. 


\section{INTRODUÇÃO}

O homem pode ser imaginado, num aspecto mais abrangente, como um cientista imperfeito, inserido em uma realidade social. Esses sujeitos e seus laços se estabelecem em um território na busca de qualidade de vida.

A qualidade de vida depende em parte, da garantia de saúde e controle ou cura das doenças. No tocante ao fenômeno saúde e doença, não se pode desagregar as teorias e as práticas de saúde dos aspectos da cultura do meio social. Deve-se, para tanto, estar clara para qualquer pesquisador, que busque a relevância e a abordagem do fenômeno, que este possa dirigir-se genericamente a três beneficiários: a sociedade, a ciência e a escola.

É bem verdade que, quando nos referimos ao tema saúde e doença, mostra-se imperioso atentarmos para a necessidade de haver grandes modificações conceituais e práticas no que diz respeito à formação permanente dos profissionais de saúde. O direcionamento da inclusão do enfoque coletivo sobre o tema necessita abordar, em especial, o conceito ampliado de saúde com seus determinantes sociais de vulnerabilidade social e contextual que ampliam as desigualdades. (SILVA, 2020).

Neste sentido, não tem sido uma tarefa muito fácil para os profissionais da saúde e para os pesquisadores, tendo em vista que, a cada tempo, vem surgindo novas doenças, sendo elas, consideradas cada vez mais "fatais". Atualmente, a contaminação em massa pelo novo coronavírus - COVID19, na propagação pelos domínios da corporalidade (convívio e proximidade) em apertos de mão, beijo, tosse, espirro, pele e fomites (objetos inanimados contaminados) atinge todos os povos do planeta e tornou-se um desafio sanitário para a população, pesquisadores, profissionais de saúde e gestores. (ALMEIDA 2020).

Por conta disso, no Brasil, o Sistema Único de Saúde (SUS) vem a cada ano, ampliando o agrupamento das doenças, implantando novas medidas asseguradoras de prevenção e tratamento, disponibilizando orientações, tanto para a população, como também para os profissionais da saúde. O exemplo vem da publicação das novas Diretrizes Operacionais da Saúde, através da Portaria 
MS/GM no 399/2006, que institui as Diretrizes Operacionais do Pacto pela Saúde, a portaria vem sendo atualizada de acordo com as novas práticas de saúde implantadas no território brasileiro. (BRASIL, 2006).

Indubitável é que, com o aumento considerável do conhecimento humano, a ciência vem se harmonizando aos cidadãos com uma participação de fato em seu desenvolvimento, buscando dentre seus objetivos a melhoria na qualidade de vida. Ademais, dos componentes que atendem às necessidades básicas de sobrevivência e de seus desejos, sejam eles estéticos, como também clínicos. (RANIERI, 2018).

Diante do exposto torna-se pertinente questionarmos: De que maneira as novas perspectivas do conceito de saúde contribuem para a abordagem das doenças?

O objetivo do artigo é investigar as diferentes perspectivas elaboradas na abordagem do binômio saúde e doença.

O caminhado trilhado nesse estudo abrange a pesquisa qualitativa, de método exploratório.

$\mathrm{Na}$ pesquisa qualitativa o pesquisador direciona seus esforços para compreender um fenômeno, aprofundando a base de seus conhecimentos teóricos. O método exploratório, concede a oportunidade ao pesquisador de explorar e ampliar os conhecimentos, nesse caso, através do estudo e do levantamento bibliográfico sobre o tema saúde e doença. (ZANELLA, 2013).

Buscou-se desenvolver uma revisão de literatura nas bases de dados da Literatura Latino-Americana e do Caribe em Ciências da Saúde (Lilacs) e Scientific Electronic Library Online (SciELO), utilizando de forma combinada os descritores: abordagem; saúde; doença. Foram selecionados artigos científicos completos a partir da combinação das palavras-chave, escritos em português e localizados nas bases de dados.

\subsection{BREVE OLHAR SOBRE A HISTÓRIA SAÚDE E DOENÇA}

É primordial, de antemão, atentarmos ao conceito de saúde elaborado pela Organização Mundial da Saúde (OMS) em 1946, senão vejamos: "saúde é 
um estado de perfeito bem-estar físico, mental e social, e não consiste apenas na ausência de doença ou de enfermidade" da pessoa. O conceito de saúde, elaborado após a II Guerra Mundial traz uma visão além da saúde do corpo físico. Inovador, entretanto, com o passar das décadas, o conceito de saúde torna-se muito mais complexo. (GAINO et al., 2018, p. 110).

No Brasil, o atual conceito de saúde passa a considerar os princípios do Sistema Único de Saúde (SUS), integra a medicina preventiva, os cuidados de saúde e de autocuidado, paradigmas resultantes das conferências nacionais de saúde no país. (GAINO et al., 2018).

Historicamente, o conceito de saúde recebe reflexões das antigas civilizações, como a Grécia antiga onde médicos como Galeno e Celso acreditavam que saúde tratava-se do equilíbrio entre as partes elementares do corpo humano. Doença, na medicina chinesa e hindu (causa naturalizadas) acontecia no desequilíbrio das funções do corpo humano. As doenças e o desequilíbrio da saúde recebiam inúmeros determinantes e combinações casuísticas entre ambiente físico (odores fétidos), religiosos, aos astros, clima, insetos e animais, as características do vento e da água, e as estações do ano. (MORAES, 2020).

Na Idade Média, o caráter religioso concedido às doenças e o tratamento por charlatões (falsos médicos) avançam no período. A ocorrência das pestes (epidemias) recebia como justificativas a conjugação dos astros, envenenamentos das águas pelos doentes de lepra e a ocorrência de bruxarias. (LEITE, 2020).

No período do século XVIII, se inicia a prática da medicina individual e da medicina social. Uma medicina empírica que utiliza o saber e o poder para disciplinar o corpo. No final do século, o hospital passa a ser visto como um local de cura para o indivíduo doente. A medicina social assume o papel de polícia médica nas cidades, com a utopia de eliminar definitivamente as doenças do meio social. (BARROS, 2018).

O século XIX corresponde ao período da visão bacteriológica das doenças, ou seja, a doença é causada por um agente vivo e externo, precisando 
ser controlada e destruída com o uso de agentes químicos e prevenida coletivamente com o uso de imunizantes. Os estudos sobre as causa das doenças tem sua continuidade nos campos da patologia, fisiologia, clínica e microbiologia, devido ao aumento da mortalidade geral e infantil nas cidades densamente populosas a partir da Revolução Industrial. (PEREIRA, 2018).

No século $X X$, os pesquisadores ocidentais se debruçam a fragmentar minuciosamente o corpo humano e a explicar as doenças a partir de três componentes: agente, hospedeiro e meio ambiente. Henry Sigerist conceitua a expressão promoção da saúde. É o momento que a doença recebe o componente relacionado aos fatores psíquicos como um dos desencadeantes para as doenças, assim o psíquico responde ao corporal e vice-versa. 0 tratamento utiliza intervenções com agentes químicos, à prevenção acontece com intervenções ambientais, estilo de vida e avança a aplicação das vacinas. (MACHADO, 2019).

No século $X X I$, os cientistas analisam o processo saúde e doença a partir de modelos estruturais e complexos dos organismos vivos, que mantém relação entre os mesmos e se inter-relacionam com o meio ambiente onde estão inseridos. Os conceitos são construídos pelo modo de vida do indivíduo e pela comunicação com a vida, à frequência de um fenômeno comum torna-se padrão de normalidade. (SANTOS, 2017).

O conceito de saúde e doença, infere-se, que tenha muito que avançar se aperfeiçoando nas próximas décadas. Novas doenças surgem como a COVID19, outras ressurgem como a dengue, outras desaparecem como a varíola, assim as bases conceituais de saúde e doença tem relação com as várias formas de organização social do homem. Os cientistas atentam-se ao comportamento do indivíduo e do meio social em que vive, conduzindo as ações para a promoção da saúde e potencializando o poder de escolha do homem e da coletividade, frente aos condicionantes de saúde.

A transição epidemiológica, transição demográfica e a educação contribuem para as mudanças do padrão de saúde no mundo contemporâneo. 
No tratamento das doenças várias áreas do conhecimento, além da medicina, atuam nos dias atuais para que os melhores resultados sejam alcançados.

\subsection{DIFERENTES PERSPECIVAS DE ABORDAGENS}

Inegável, ao iniciarmos o tema as diferentes abordagens da saúde e doenças, citar os múltiplos os aspectos envolvidos na conceituação de saúde/doença como o fator econômico, social, cultura, educação, conhecimento, dados demográfico, acessibilidade, entre outros. O conceito saúde recebe várias conotações, aprimorando conforme se estabelece a relação entre doença, tempo e espaço.

O acesso e uso dos serviços de saúde estabelecem relações conforme Assis e Jesus (2012), relacionadas aos aspectos individuais: predisponentes (problema); capacitantes (renda e oferta de serviços de saúde) e diagnósticas (necessidades a partir do diagnóstico).

A Saúde Pública se organiza em torno da doença, uma visão de estudo das disciplinas médicas e da Antropologia Social. A Antropologia Médica e a Epidemiologia se preocupam com a doença, assim como sua evolução e desfecho dos casos de estudos.

A visão da epidemiologia é a doença, estuda a incidência e a prevalência das doenças a partir do diagnóstico. A notificação de um caso demanda investigação, acompanhamento, desfecho e encerramento. Dados quantitativos abastecem os sistemas de informação, podendo ser acessados por cidadãos, profissionais de saúde e gestores. A realidade dessa ciência é voltada integralmente para as mudanças da sociedade, buscando conhecer marcos histórico e seus efeitos práticos no conceito de risco. (ASSIS; JESUS, 2012; BOSI, 2015).

As descrições pragmáticas e quantitativas das doenças, realizada pela Epidemiologia envolve uma complexidade de fatores como perfil socioeconômico, dados de morbidade e mortalidade, sociológicos, econômicos, ambientais e culturais. Além de proceder ao descarte os casos suspeitos e falsos positivos. (SANTOS et al., 2017). 
Um dos problemas da abordagem da epidemiologia são a subnotificação de casos, a este respeito, alguns pontos podem ser citados: profissionais de saúde que "esquecem" de notificar o caso (suspeito ou confirmado), muitos profissionais da rede privada de saúde colaboram na subnotificação e os grupos sociais em vulnerabilidade social não procuram os serviços de saúde ou procuram menos ficando a margem do trabalho da epidemiologia. (SANTOS et al., 2017).

O conceito de risco em saúde para a epidemiologia é diferente em cada área territorial de atendimento dos serviços de saúde, precisando ser construído a partir do diagnóstico sanitário e social local. Em um mesmo município, podemos encontrar desigualdades sociais e demográficas, área com concentração de idosos com diagnóstico de doenças crônicas (diabetes) e em outra área concentração de crianças com diagnóstico de desnutrição (dieta pobre em nutrientes). (ARAÚJO, 2014; BOSI, 2015).

Outro polo é identificar e avaliar as demandas reprimidas e os focos de exclusão de atendimento em saúde. A abordagem inicia na Atenção Primária à Saúde a partir do diagnóstico epidemiológico e demográfico, reduzindo os casos de doenças/doentes que possam evoluir e serem encaminhados aos serviços públicos hospitalares na atenção secundária. (SANTOS et al., 2017).

A Antropologia e a Epidemiologia vêm contribuindo para uma nova perspectiva de saúde e doença na cultura ocidental, além da visão biologista. $O$ avanço dos estudos sobre as doenças e de novos conceitos de saúde apresentam a sinergia entre indivíduo e cultura, a integração dos componentes histórico e social, e as experiências individuais (valores e costumes), assumindo papel importante na abordagem dos problemas de saúde. (LANGDON, 2014).

Destaque para a reflexão de Landgton et al. 2012, para as autoras,

O desenvolvimento do conhecimento é dependente, em grande medida, das instituições onde as pesquisas são conduzidas. "Forças estruturais externas e internas" podem estimular ou retardar seu avanço, já que afetam a troca criativa de ideias. (LANDGTON et al. 2012, p. 53). 
A Antropologia busca estudar o homem na sua diversidade cultural. $\mathrm{O}$ homem contemporâneo tem relação com o tempo da época em que vive, adere ou mantem-se distante das transformações do seu tempo. A globalização e a saúde global trouxeram avanços e inquietações na área do conhecimento sobre saúde e doenças, são fatores que interferem nas escolhas, na adaptação e na vida do homem. (IRIART; CAPRARA, 2011).

Como ciência a Antropologia se debruça nas relações de poder que acontecem nas relações sociais, elas também aparecem nas relações entre os usuários do sistema de saúde e os profissionais de saúde. Para Gusmão (2015), as relações de poder emergem entre os desiguais em um processo de estranhamento (de longe e de perto) e construídas na sociedade. A autora sugere os seguintes processos:

\footnotetext{
- Homogeneização (objetivo de agentes e agências que operam o tecido social e implicam um campo de poder)

- Contradição (socialização/sociabilidades construídas nas relações sociais entre sujeitos diversos e na gestão dos interesses coletivos); - Conflito (de classe e de outra natureza, tais como raça, cor, gênero, etc...). (GUSMÃO, 2015, p. 25).
}

A Antropologia da Saúde aponta os limites do estado de saúde de uma determinada população, avaliando aspectos relacionados aos hábitos e estilo de vida, a vida social e cultural do indivíduo. (LARRUBIA et al., 2019).

A ciência, também identifica as fragilidades da tecnologia médica, em relação às tentativas de mudanças permanentes de hábitos inadequados e afastamento de fatores de risco da população. Segundo, Iriart e Caprara (2011) "é fundamental levar em conta os fatores culturais contextualizando-os histórica e politicamente".

A responsabilidade individual da própria saúde e do autocuidado na prevenção de fatores de risco às doenças, articulam os campos da política, cultura, economia e sociedade, na ideia básica que o indivíduo é o personagem principal e responsável entre o equilíbrio entre saúde e doença. (GIMENES, 2013). Os corpos se modificam, assim como o pensamento social, à medida que 
o conhecimento em saúde avança e implica em um novo conceito para a humanidade.

A Antropologia Médica assume um papel importante como norteador de novos conceitos construídos com uma visão crítica do homem e do binômio saúde - doença, analisando a relação da vida (biologia, psíquica, social, cultura, familiar, individual) e o meio ambiente.

Muitos dilemas éticos surgem na Antropologia Médica na contemporaneidade, como as redefinições da saúde e doença ligadas aos avanços da biologia molecular, da genômica e as biotecnologias no tratamento das doenças. A globalização mostra um mapa de desigualdades sociais, quando analisamos as populações em suas fronteiras territoriais e seus determinantes de saúde, tornando urgente a intervenção da antropologia social e médica. (IRIART; CAPRARA, 2011).

No campo da cultura a antropologia estuda a formação social, utilizando recursos e técnicas de pesquisa, compreendem as relações estabelecidas, consequências internas e externas. A cultura tem como característica ao mesmo tempo estar estável e ser mutável, tem ação seletiva de possibilidades e limites para se fragmentar e reconstruir. O mesmo acontece om a saúde e a doença. (SANTOS; REGERT, 2018).

\section{EDUCAÇÃO EM SAUDE}

Educação é o método preventivo principal para que a saúde aconteça e as doenças mantenham-se distante do indivíduo e da coletividade.

Educação em saúde é declaradamente enfatizada por muitos autores, como a estratégia que possibilita o planejamento de ações para promover saúde na atenção primária, a partir do diagnóstico situacional dos indivíduos no território de atendimento dos profissionais de saúde que atuam na saúde coletiva. (SANTOS; RIGOTTO, 2010; KLEBA et al. 2011; KLEBA et al., 2015).

As ações desenvolvidas pelos profissionais de saúde exercem influência na tomada de atitudes dos indivíduos e nos processos de mudanças para alcançar a promoção da saúde. As atividades podem envolver adoção de hábitos 
alimentares saudáveis, exames de rotina para prevenção de doenças, prática de exercícios físicos regulares, adequação do meio ambiente, eliminação de hábitos que coloquem a saúde em risco e até mesmo a forma correta de utilização dos serviços de saúde. (CÂMARA et al., 2012).

A educação voltada para a saúde busca, ainda, desenvolver no indivíduo o encargo do autocuidado e a responsabilidade por suas escolhas em relação à sua saúde, além do senso de coletividade, onde as escolhas de um cidadão refletem em todos os membros da sociedade. Aproximar os conhecimentos científicos da população oferece aos profissionais da saúde, a certeza de conferir um leque de escolhas para uma vida saudável. (CÂMARA et al., 2012).

Os modelos clássicos de educação em saúde prezam pelas ações individuais de diagnósticos por exames, palestras, vacinação e terapêuticas medicamentosas a grupos em situação de vulnerabilidade em saúde. As equipes de saúde da família devem estar cientes do seu papel educacional e utilizar filtros (linguagem, religião, cultura, conhecimento técnico-cientifico, entre outras) para prestar assistência ao indivíduo, à família e à comunidade. (VIANNA, 2015).

A base da educação em saúde é indissociável da ciência e da condição humana, a aprendizagem acontece inerente da prática social e da vida do indivíduo, o que altera são os métodos. Os processos educativos se desenvolvem além da escola e avançam pelos tecidos sociais, independente das múltiplas e diversas culturas. (GUSMÃO, 2015).

A dialógica e a etnografia são eficientes recursos na educação em saúde. Os educadores em saúde necessitam aprofundar seus conhecimentos em ciências sociais e comunicação para compreenderem os comportamentos desiguais (idade, gênero, classe, cultura, entre outros) do indivíduo e do coletivo em relação à saúde. (MARINEZ-HERNÁEZ, 2010).

Outro aspecto é evitar o monólogo e o unidirecionamento no processo educacional, atualizando o conhecimento e a imagem do meio social onde os profissionais de saúde atuam (expressões, valores, hábitos, etc.), evitando o preconceito que o usuário do serviço de saúde nada sabe, assim como a 
hierarquização (do mais para o menos) e a verticalização (de cima para baixo) educacional do profissional de saúde sabe tudo. (MARINEZ-HERNÁEZ, 2010).

\section{CONSIDERAÇÕES FINAIS}

Ao longo do artigo, diferentes perspectivas de abordagem da saúde e doença foram apresentadas, trazendo assim uma ideia da evolução dos conceitos dessas palavras.

Inicialmente apresentamos alguns aspectos sobre a evolução do conceito de saúde, como a visão dos médicos da Grécia antiga que saúde atendia ao equilíbrio das partes elementares do corpo humano, a influência multifatorial na construção dos conceitos (inseto, religião, magia, envenenamento, etc.) aos modelos complexos (genético, psíquico, estilo de vida, social, etc.). Nas primeiras décadas do século XXI, a saúde é analisada além dos modelos estruturais complexos, observando suas relações com o organismo vivo e o meio ambiente onde se insere.

A imagem construída de saúde e doença com a ajuda da Antropologia e da Epidemiologia, nos possibilita enxergar o caminho percorrido nos diferentes tecidos sociais pelos indivíduos e pelos profissionais da saúde. O estudo da formação social é importante para a utilização dos recursos de saúde e técnicas de pesquisa corretas, além do conhecimento dos envolvidos sobre o processo das relações sociais internas e externas.

A educação em saúde reafirma a importância do diálogo no contexto educacional. Os envolvidos, principalmente os profissionais da saúde devem estar empossados de conhecimento técnico e social, além de princípios das ciências sociais e da comunicação, reconhecimento da realidade do usuário dos serviços de saúde, desprovidos de preconceitos e abertos para a abordagem diferenciada em educação em saúde.

Os estudos precisam avançar para apresentar e contextualizar a compreensão das condições ideias de abordagem em educação em saúde e doença. 


\section{REFERÊNCIAS}

Almeida, g. Muito além da perda de libido. 2020. In: Iole, a.; stampa i.; gomes, r. L. R.(org.). Para além da quarentena: reflexões sobre crise e pandemia. São paulo: puc, 2020. P. 191-199. Disponível m: https://morula.com.br/wpcontent/uploads/2020/06/paraalemdaquarentena.pdf. Acesso em: 10 maio de 2021.

Alves, p. C., rabelo, m. C. (org.). Antropologia da saúde: traçando identidade e explorando fronteiras. Rio de janeiro: editora fiocruz,1998.

Araújo, jandira aureliano. Análise de demandas de saúde ambiental de comunidade vulnerdas e das respostas da política de saúde local. 2014. Tese (doutorado em ciências) - centro de pesquisas aggeu magalhães, fundação osvaldo cruz, recife, 2014.

Assis, m. M. A.; jesus, w I. A. Acesso aos serviços de saúde: abordagens, conceitos, políticas e modelo de análise. Cienc. Saúde coletiva, v. 17, n. 11, p. 2865- 2875, 2012. Disponível em: https://www.scielo.br/j/csc/a/qlyl8v4vlzqp6s5fpr8mlgp/?lang=pt\&format=html. Acesso em: 10 set. 2021.

Barros, j. R. A cidade medicalizada. Prometeus - filosofia, ano 11, n. 26, p. 179-192, 2018.2 Disponível em: https://seer.ufs.br/index.php/prometeus/article/view/6560. Acesso em: 20 maio de 2021.

Bosi,m. L. M. Problematizando o conceito de risco em diretrizes éticas para pesquisas em ciências humanas e sociais na saúde coletiva. Ciênc. Saúde coletiva, v. 20, n. 9, p. 2675-2682, 2015. Disponível em: https://www.scielosp.org/pdf/csc/2015.v20n9/2675-2682/pt. Acesso em: 10 ago. 2021.

Brasil. Ministério da saúde. Portaria no 399, de 22 de fevereiro de 2006. Divulga o pacto pela saúde 2006 - consolidação do sus e aprova as diretrizes operacionais do referido pacto. Brasília: ministério da saúde, 2006.

Camâra, a. M. C. S.; melo, v. L. C.; gomes, m. G. P.; pena, b. C.; oliveira, k. M.; moraes, a. P. S.; coelho, g. R.; victorino, I. R. Percepção do processo saúdedoença: significados e valores da educação em saúde. Revista brasileira de educação médica, v. 36, n. 1 supl., p. 40-50, 2012. Disponível em: https://www.scielo.br/j/rbem/a/kr5x5x4qznwyckmdfxdbqfn/abstract/?lang=pt. Acesso em: 10 jul. 2021.

Carrapato, p.; correia, p.; garcia, b. Determinante da saúde no brasil: a procura da equidade na saúde. Saúde soc., v. 26, n. 3, p. 676-689, 2017. Disponível em: 
https://www.scielosp.org/pdf/sausoc/2017.v26n3/676-689/pt. Acesso em: 20 jul. 2021.

Gaino, I. V.; souza, j.; cirineu, c. T.; tulimosky, t. D. O conceito de saúde mental para profissionais de saúde: um estudo transversal e qualitativo. Smad, rev. Eletrônica saúde mental álcool drog., v. 14, n. 2, p. 108-116, 2018. Disponível em http://pepsic.bvsalud.org/scielo.php?script=sci_arttext\&pid=s1806$69762018000200007 \&$ Ing=pt\&nrm=iso. Acesso em 30 nov. 2021.

Gimenes, g. F. Usos e significados da qualidade de vida nos discursos contemporâneos de saúde. Trab. Educ. Saúde, rio de janeiro, v. 11, n. 2, p. 291318, 2013. Disponível em:

Https://www.scielo.br/j/tes/a/4btkgrdczrxyzcsc7j5ks4h/?format=pdf\&lang=pt. Acesso em: 26 out. 2021.

Gusmão, n. M. M. Antropologia e educação: um campo e muitos caminhos. Linhas críticas, v. 21, n. 44, p. 19-37, 2015. Disponível em: https://www.redalyc.org/pdf/1935/193538270003.pdf. Acesso em: 20 maio de 2021.

Iriart, j. A. B.; caprara, a. Novos objetos e novos desafios para a antropologia da saúde na contemporeneidade. Physis revista de saúde coletiva, v. 21, n. 3, p. 853-863, 2011. Disponível em: https://www.scielo.br/j/physis/a/j3h3zmlskrz9qhvbdk7fhfh/abstract/?lang=pt. Acesso em: 10 set. 2021.

Langdon, e. J. Os dialógos da antropologia com a saúde: contribuições para as políticas públicas. Ciênc. Saúde coletiva, v. 19, n. 4, p. 1019-1029, 2014. Disponível em: https://www.scielosp.org/pdf/csc/2014.v19n4/1019-1029/pt. Acesso em: 26 maio 2021.

Langdon, e. J.; follér, m. L.; maluf, s. W. Um balanço da antropologia da saúde no brasil e seus diálogos com as antropologias mundiais. Anuário antropológico, v. $37, \quad$ n. $1, \quad$ p. $51-89,2018$. Disponível em: https://periodicos.unb.br/index.php/anuarioantropologico/article/view/7252.

Acesso em: 30 out. 2021.

Larrubia, b. C.; freitas junior, n. E.; freitas, i. M. Antropologia da saúde e doença: contribuições para os serviços públicos de saúde. Revista científica multidisciplinar núcleo do conhecimento, ano 4, ed. 8, v. 4, p. 5-28, 2019. Disponível em: https://www.nucleodoconhecimento.com.br/administracao/servicos-publicos. Acesso em: 16 maio 2021.

Leite, g. Conceito de saúde na contemporaneidade. Blog jornal jurid, 2020. Disponível em: https://www.jornaljurid.com.br/colunas/gisele-leite/conceito-desaude-na-contemporaneidade. Acesso em: 30 set. 2021. 
Kleba, m. E.; duarte, t. F.; romanini, a.; cigognini, a.; althaus, i. R. Estimativa rápida participativa como ferramenta de diagnóstico na estratégia saude da família. Revista grifos, n. 38/39, p. 159-177, 2015.

Kleba, m. E.; krauser, i. M.; vendruscolo, c. O planejamento situacinal no ensino da gestão em saúde da família. Texto contexto enferm., v. 20, n. 1, p. 184-193, $2011 . \quad$ Disponível em: https://www.scielo.br/j/tce/a/mj874kdgtwvjm9f4lwpcvcr/?lang=pt. Acesso em: 20 maio de 2021.

Machado, rosangela fátima de oliveira. Territoralização da saúde: determinantes ambientais e o cotidiano das equipes de saúde da família - lagarto (se). 2019. Tese (doutorado em geografia) - programa de pós-graduação em geografia, pró-reitoria de pós-graduação e pesquisa, universidade federal de sergipe, $2019 . \quad$ Disponível em: https://ri.ufs.br/bitstream/riufs/11030/2/rosangela fatima oliveira machado.pdf. Acesso em: 20 nov. 2021.

Martínez-hernáez, a. Dialógica, etnografia e educação em saúde. Rev saúde pública, v. 44, n. 3, p. 1-7, 2010. Disponível em:

Https://www.scielo.br/j/rsp/a/k4lfcy3gql8lqpd73txfzdb/abstract/?lang=pt acesso em: 20 maio de 2021.

Moraes, m. C. V. O corpo nas tradições orientais. In: bassani, m. A. Bassani (org.). Diálogos entre psicologia, espiritualidade e meio ambiente: o sagrado em perspectiva. São paulo: educ, 2020. P. 231-244.

Pereira. P. F.; souza, c. T. V.; hora, d. L.; possas, c. A.; menezes, r. C. O ensino da patologia e sua influência na atuação de patologistas e infectologistas no rio de janeiro. Revista basileira de educação médica, v. 42, n. 1, p. 214-223, 2018. Disponível em: https://www.scielo.br/j/rbem/a/jdzqbbhtrdn4hzzwmqh8ytg/?lang=pt. Acesso em: 20 maio de 2021.

Ranieri, n. B. S.; alves, a. L. A. Direito à educação e direitos na educação em perspectiva interdisciplinar. São paulo: universidade de são paulo (usp), 2018.

Santos, s. V. M.; macedo, f. R. M.; resck, z. M. R.; sanches, r. S.; nogueira, d. A.; terra, f. S. Características socieconômicas, epidemiológicas e laborais de profissionais de enfermagem hospitalar. Revista de enfermagem do centrooeste mineiro, v. 7, e1391, p. 1-12, 2017. Disponível em: http://www.seer.ufsj.edu.br/index.php/recom/article/viewfile/1391/1567. Acesso em: 20 maio de 2021.

Santos, a. M.; regert, r. Antropologia educacional: a consolidação de uma subárea epistemológica. Vivência revista de antropologia, v. 1, n. 52, p. 214- 
225, 2018. Disponível em: https://periodicos.ufrn.br/vivencia/article/view/13190. Acesso em: 10 mar. 2021.

Santos, a. L.; rigotto, r. M. Território e territorialização: incorporando as relações de produção, trabalho, ambiente e saúde na atenção básica à saúde. Trab. Educ. Saúde, v. 8, n. 3, p. 387-406, 2010. Disponível em: https://www.scielo.br/j/tes/a/h5btbjtgvqzgsxkvnrtkphp/?lang=pt. Acesso em: 26 maio de 2021.

Silva, d. N. Determinantes socias da vulnerabilidade à covid-19: proposta de um esquema teórico - parte ii. Unifesspa, p. 1-10, 2020. Disponível em: https://acoescovid19.unifesspa.edu.br/images/artigo_-_parte_2_-_completo.pdf. Acesso em: 22 jul. 2021.

Vianna, I. A. C. Processo saúde-doença. São paulo: unifes, 2015.

Zanella, I. C. H. Metodologia de pesquisa. 2. Ed.reimp. Florianópolis: departamento de ciências da administração/ufsc, 2013. Disponível em: Http://arquivos.eadadm.ufsc.br/eadadm/uab_2014_2/modulo_1/metodologia/ma terial_didatico/livrotextometodologiadapesquisa.pdf. Acesso em 18 out 2021. 